\title{
Stability Analysis of a MEMS Acceleration Sensor
}

\author{
Heiko Wolfram*, Wolfram Dötzel \\ Chemnitz University of Technology, Faculty of Electrical Engineering and Information Technology \\ Department of Microsystems and Precision Engineering, D-09107 Chemnitz, Germany
}

\begin{abstract}
The electrostatic actuation with its several advantages is the main principle for MEMS. One major drawback is the nonlinear behavior, which results into instability, known as the electrostatic pull-in effect. This effect might also push a closed-loop configuration into instability and thus makes a linear time-invariant control inapplicable to the system. The paper investigates the stability of an acceleration sensor in closed-loop operation with this setting. A simplified controller adjustment gives a first insight into this topic. Practical implementations saturate on the quantizer's full-scale value, which is also considered in the stability analysis. Numerical phase-plane analysis verifies the stability and shows further surprising results.
\end{abstract}

\section{INTRODUCTION}

Micro-electro-mechanical systems (MEMS) play an important role for realization of sensor/actuator systems. MEMS are small, very compact, have a simple and robust layout and are producible in large quantities. Another advantage is the technology, which can directly be obtained from the microelectronics and thus makes the integration of the electronics possible. Also, the electrostatic principle can be widely used to measure pressure, sonic waves, fill level, vibration, acceleration, etc.

There are, however, some limitations such as packaging, cross-talk related problems, the MEMS mechanical limitation and in the particular of control engineering, the strong nonlinearity of the vertical electrostatic field component and the nonlinear fluid damping. Therefore, an intensive analysis of the system stability might be required.

Control systems were widely applied to MEMS to improve the system's dynamical behavior. A proportional control [1] and sigma-delta converter [2], [3], [4] were used to control an accelerometer. Lead-lag filters are partly taken to enhance the system behavior. A phase lead-lag controller in combination with a second-order low-pass filter [5], [6] was successfully applied to micro-mirror systems. A Kalman filter state observer, incorporated with state feedback, containing integral action [7], found its application for microactuators. Model reference adaptive control systems (MRAC) were successfully applied to micro-mirrors [8] and gyroscopes [9], [10]. The nonlinear approach of sliding-mode control [11] was used to control a micro-mirror, as well as a model reference based neural network [12] to control an accelerometer.

Unfortunately, only a few efforts were made to completely analyze the system from control perspectives. Mostly, the MEMS were assumed to be stable $\mathrm{PT}_{2}$-Systems (e.g. in [1],

*Corresponding author: Tel.: +49371 531-3274; Fax: +49371 531-3259;

Email: heiko.wolfram@e-technik.tu-chemnitz.de
[13]) without doing further analysis. This assumption could be fatal for an operation near the pull-in point, where the electrostatic force is equal to the spring force.

The stability, which is displacement and control signal dependent, is difficult to prove. Assumptions were usually made to operate the system in the stable region with neglecting the nonlinear behavior. Stability was checked in [14] with calculating the phase and gain margins for a deflection dependent linearization. A similar approach can be found in [15], where the root locus at maximal (beneath the pull-in point) and minimal displacement is used as the stability criteria.

Our paper follows Lyapunov's approach to prove the stability of a special parameter setting in a far greater region. Lyapunov's indirect method ensures the stability close to the equilibrium state, where the direct method shows local stability in a wider area. Phase plane analysis proves the stability and offers an even further stable region.

\section{StRUCTURE AND DESIGN}

A major advantage of the electrostatic principle is the unification of the sensor and actuator effect in the same field area. This makes an extra component needless and favors a closed-loop operation.

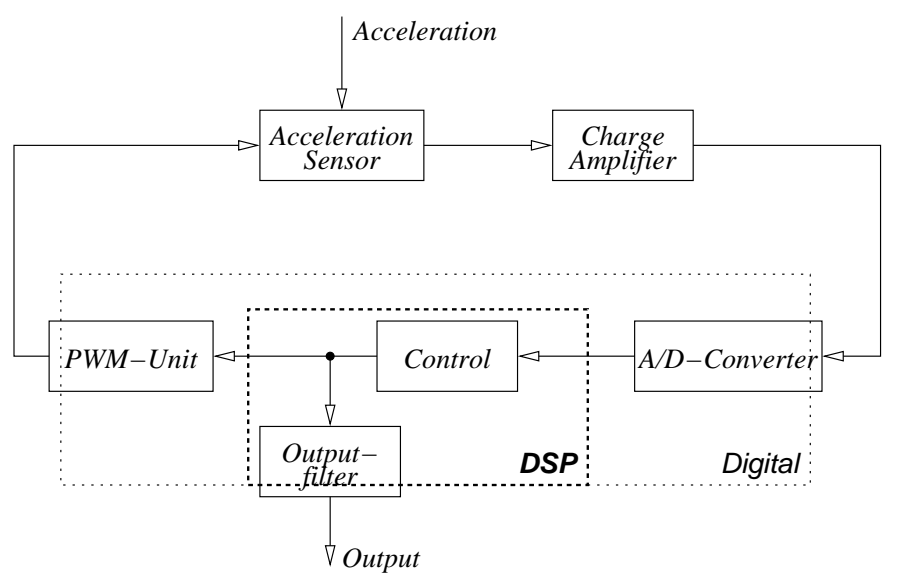

Fig. 1. Block diagram of the system

The system configuration is shown in Fig. 1. The digital signal processor (DSP) realizes the control system and further signal processing, like bias and nonlinearity correction, and filtering.

The applied charge amplifier, being the measurement system, has two tasks. Firstly, it transforms the charge, which is a measure of the capacitance, into a proportional voltage. 
Secondly, the switched mode operation achieves the seismic mass positioning with a proper duty cycle of the pulse width modulated (PWM) signal.

The sensor was fabricated in bulk-micromachining technology and consists of a glass-silicon-glass sandwich structure. This configuration minimizes the parasitic capacities, arising mainly from the sensor frame, and thus reduces the electronic effort in the detection circuit noticeably. Fig. 2(a) shows the schematic chip configuration.

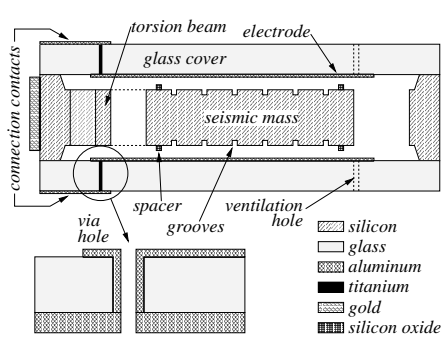

(a) Schematic configuration

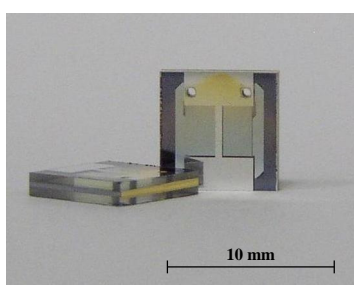

(b) Photo
Fig. 2. The sensor chip

The seismic mass is suspended by two vertical torsion beams. The grooves on the mass surface noticeably reduce the air damping in the gap without influencing the electrostatic field too much. The $\mathrm{SiO}_{2}$-spacer close to the edges of the seismic mass prevent the electrical contact with the outer electrodes.

Drill holes in the glass cover provide the equalization to ambient pressure. Ambient pressure is the reason for a simple $\mathrm{PT}_{1}$-model, which can be found in [16]. It also minimizes deformations during the bonding process and provides approximate equal dynamics for all sensor samples.

\section{Control Design}

A very easy method to tune the control is the use of Bode's Ideal Loop Transfer Function [17]. The open loop transfer function has in that case a slope of $-20 \mathrm{~dB} /$ decade over the whole frequency range

$$
\operatorname{abs} L(j \omega)=\frac{\omega_{c}}{\omega},
$$

with the crossover frequency $\omega_{c}$, which is the desired bandwidth of the open-loop function. This method might not be propitious for a good design, but gives a first insight into the system limitations and simplifies the further calculations.

\section{Stability Analysis}

Lyapunov's indirect method proves with the linearization of the nonlinear system the asymptotic stability at the equilibrium state $\mathbf{x}=\mathbf{0}$. All the eigenvalues of the system matrix must lie in that case strictly in the left-half complex plane (LHP). But there is no guaranteed local stability in a wider area for the nonlinear control system.
Consider the autonomous system

$$
\dot{\mathbf{x}}=\mathbf{f}(\mathbf{x})
$$

which can be evaluated with the use of a PWM and charge amplifier gain $k_{p w m}$ and $k_{a}$, the difference capacitance $\Delta C(\varphi)$, the electromechanical moment $M_{e l}(\varphi, u)$, the $\mathrm{PT}_{1}$-model $G_{\text {mech }}=k_{p} /\left(T_{1} s+1\right)$ and the applied PI-control $K=$ $\left(b_{1} s+b_{0}\right) / s$ to

$$
\left[\begin{array}{c}
\dot{\varphi} \\
u
\end{array}\right]=\left[\begin{array}{c}
\frac{1}{T_{1}}\left(-\varphi+k_{p} M_{e l}(\varphi, u)\right) \\
k_{p w m} k_{a}\left(b_{0} \Delta C(\varphi)+\ldots\right. \\
\left.\frac{b_{1}}{T_{1}} \frac{\mathrm{d} \Delta C(\varphi)}{\mathrm{d} \varphi}\left(-\varphi+k_{p} M_{e l}(\varphi, u)\right)\right)
\end{array}\right] .
$$

The Jacobian matrix of the system is then given by

$$
\begin{aligned}
\frac{\partial \mathbf{f}}{\partial \mathbf{x}} & =\left[\begin{array}{ll}
\frac{\partial f_{1}}{\partial x_{1}} & \frac{\partial f_{1}}{\partial x_{2}} \\
\frac{\partial f_{2}}{\partial x_{1}} & \frac{\partial f_{2}}{\partial x_{2}}
\end{array}\right] \\
& =\left[\begin{array}{cc}
-\frac{1}{T_{1}}+\frac{k_{p}}{T_{1}} \frac{\partial M_{e l}}{\partial \varphi} & \frac{k_{p}}{T_{1}} \frac{\partial M_{e l}}{\partial u} \\
\frac{\partial f_{2}}{\partial x_{1}} & \frac{b_{1} k_{p} k_{p w m} k_{a}}{T_{1}} \frac{\mathrm{d} \Delta C(\varphi)}{\mathrm{d} \varphi} \frac{\partial M_{e l}}{\partial u}
\end{array}\right]
\end{aligned}
$$

with

$$
\begin{aligned}
\frac{\partial f_{2}}{\partial x_{1}}=k_{p w m} k_{a} \frac{\mathrm{d} \Delta C(\varphi)}{\mathrm{d} \varphi}\left[b_{0}+b_{1} \frac{\partial f_{1}}{\partial x_{1}}\right]+\ldots \\
b_{1} k_{p w m} k_{a} \frac{\mathrm{d}^{2} \Delta C(\varphi)}{\mathrm{d} \varphi^{2}} \frac{\mathrm{d} \varphi}{\mathrm{d} t}
\end{aligned}
$$

With using the linearization gains $k_{c}$ of $\Delta C(\varphi), k_{\varphi}$ and $k_{u}$ of $M_{e l}(\varphi, u)$ and realizing, that the point $\varphi=0$ of function $\Delta C(\varphi)$ is an inflection point, the linearized model at the equilibrium point gives

$$
\frac{\partial \mathbf{f}}{\partial \mathbf{x}} /_{\mathbf{x}=\mathbf{0}}=\left[\begin{array}{cc}
\frac{1}{T_{1}}\left(-1+k_{p} k_{\varphi}\right) & \frac{k_{p} k_{u}}{T_{1}} \\
\frac{\partial f_{2}}{\partial x_{1}} / \mathbf{x}=\mathbf{0} & \frac{b_{1} k_{p w m} k_{a} k_{c} k_{p} k_{u}}{T_{1}}
\end{array}\right]
$$

with

$$
\frac{\partial f_{2}}{\partial x_{1} / \mathbf{x = 0}}=k_{p w m} k_{a} k_{c}\left[b_{0}+b_{1} \frac{\partial f_{1}}{\partial x_{1} / \mathbf{x}=\mathbf{0}}\right] .
$$

The PI-control can be tuned with using the Ideal Loop Transfer Function (1) to

$$
\begin{aligned}
& b_{0}=-\frac{\omega_{c}}{k_{p} k_{p w m} k_{a} k_{c} k_{u}}\left(-1+k_{p} k_{\varphi}\right) \\
& b_{1}=-\frac{\omega_{c} T_{1}}{k_{p} k_{p w m} k_{a} k_{c} k_{u}} .
\end{aligned}
$$

Substituting this values into the linearized model (5) results into

$$
\frac{\partial \mathbf{f}}{\partial \mathbf{x}} /_{\mathbf{x}=\mathbf{0}}=\mathbf{A}=\left[\begin{array}{cc}
\frac{1}{T_{1}}\left(-1+k_{p} k_{\varphi}\right) & \frac{k_{p} k_{u}}{T_{1}} \\
-\frac{2 \omega_{c}}{k_{p} k_{u}}\left(-1+k_{p} k_{\varphi}\right) & -\omega_{c}
\end{array}\right]
$$


Solving (7) for the eigenvalues gives

$$
\begin{gathered}
\lambda_{1 / 2}=\frac{\frac{1}{T_{1}}\left(-1+k_{p} k_{\varphi}\right)-\omega_{c}}{2} \pm \ldots \\
\sqrt{\frac{\left(\frac{1}{T_{1}}\left(-1+k_{p} k_{\varphi}\right)-\omega_{c}\right)^{2}}{4}-\frac{\omega_{c}}{T_{1}}\left(-1+k_{p} k_{\varphi}\right)},
\end{gathered}
$$

which have to be located strictly in the LHP. That is

$$
\omega_{c}>\frac{1}{T_{1}}\left(-1+k_{p} k_{\varphi}\right)=p_{1}^{e l}\left(u_{b}\right) .
$$

This is actually clear, since the presence of an unstable pole puts a lower limit on the system bandwidth (see e.g. [18], [19]).

Unfortunately a valid inequality still not proves the stability in a wider area for the nonlinear system, which will be considered next in an example.

$$
\begin{aligned}
& \text { V. EXAMPLE } \\
& \text { Consider the plant model }{ }^{1} \\
& G_{\text {mech }}=\frac{0.2249}{s+52.63}, \quad k_{\text {corr }}=0.45,
\end{aligned}
$$

which is evaluated from a practical sample, where $k_{\text {corr }}$ indicates the correction gain in the open loop.

Finding an energy function $V(\mathbf{x})$ for Lyapunov's direct method is not a trivial task. The function $V(\mathbf{x})$ is positive definite and its time derivative along any state trajectory of system (2) is negative semi-definite. Only in that case the energy does not increase. Very promising is the use of the function candidate

$$
V(\mathbf{x})=\frac{1}{2} \mathbf{x}^{\mathrm{T}} \mathbf{P} \mathbf{x}, \quad \mathbf{P A}+\mathbf{A}^{\mathrm{T}} \mathbf{P}=-\mathbf{I}
$$

where the quadratic matrix $\mathbf{P}$ must be positive definite.

The time derivative of the Lyapunov function (Fig. 3(c)) is in fact in a wide range of equal energies (Fig. 3(b)) positive definite and therefore local asymptotical stable. The function leaves the negative values (red area) only in the area at maximal displacement, where no conclusion about stability can be drawn.

Some similar deduction can be made by inspecting the eigenvalues of the linearized Jacobi matrix (4) at the actual operating point. However, the unstable area is obviously greater spread.

Practical realizations limit the measurement signal due to the theoretically infinite difference capacitance. Fig. 4 shows the stability losses of this configuration. The output of the amplifier saturates at $5 \mathrm{~V}$, where the tangent hyperbolic (Fig. 4(a) $)$ is used as a smooth approximation of the saturation function.

The further restricted stable area can be very clearly seen in Fig. 4(b). This is actually clear, since the observation of the

${ }^{1}$ Further parameters are: seismic mass $(l \times w \times d): 3.5 \mathrm{~mm} \times 3.5 \mathrm{~mm} \times$ $300 \mu \mathrm{m}$; gap width: $3 \mu \mathrm{m}$; operating voltage: $20 \mathrm{~V}$; amplifier feedback capacitance: $47 \mathrm{pF}$

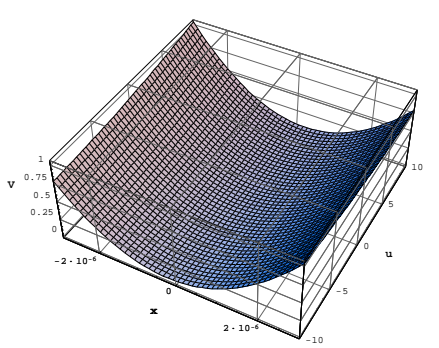

(a) Lyapunov function

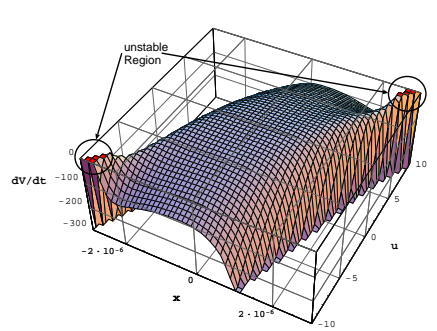

(c) Time derivative along states

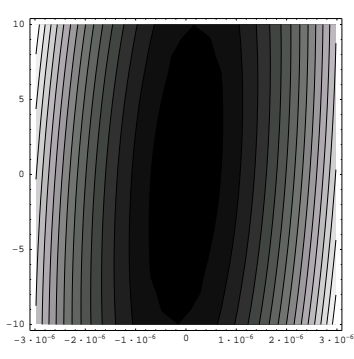

(b) Contour diagram of equal energies

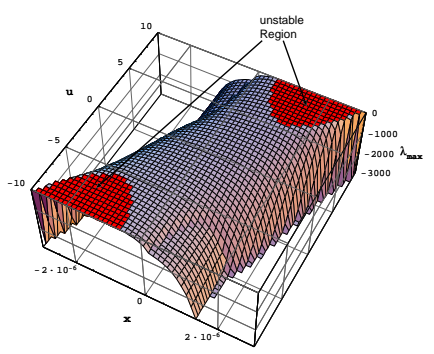

(d) Maximal eigenvalue
Fig. 3. Stability of the accelerometer with a system bandwidth of $\omega_{c}=$ $2 \pi 200 \mathrm{~Hz}$

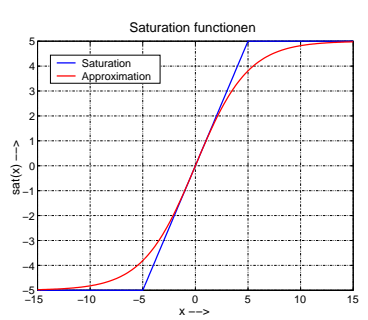

(a) saturation function

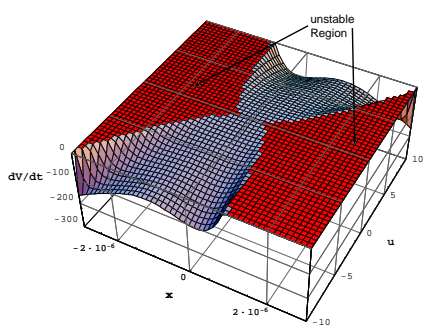

(b) Time derivative along states
Fig. 4. Stability at output amplifier saturation

seismic mass displacement is limited to some small region. Therefore, a trade-off between stability and sensitivity has to be found in practical realizations.

A further analysis is done with evaluating the phase portraits numerically [20] with different parameter adjustments. Fig. 5(a) shows a very surprising result - The system is asymptotically stable in the whole deflection area. The expected limited stability at output saturation is shown in Fig. 5(b). The actual unstable region ${ }^{2}$ is smaller compared to the calculation in Fig. 4(b).

Another configuration is shown in Fig. 5(c), where the gain crossover frequency $\omega_{c}$ is almost as low as the pole location. This results into a gain and phase margin close to zero and also

\footnotetext{
${ }^{2}$ The hatched red area marks the unstable region in the plots
} 


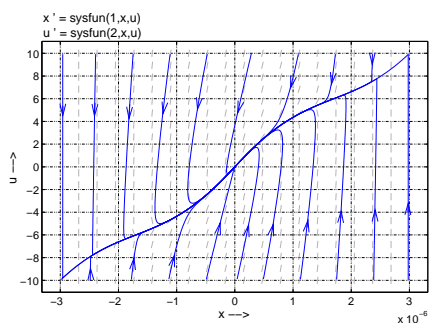

(a) System bandwidth $\omega_{c}=$ $2 \pi 200 \mathrm{~Hz}$

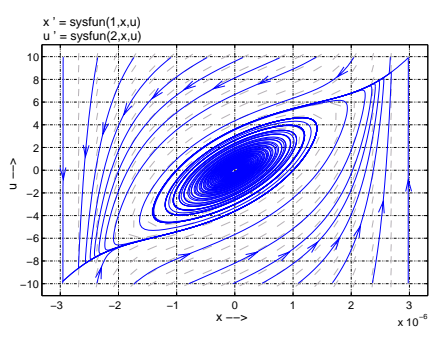

(c) System bandwidth $\omega_{c}=$ $2 \pi 21 \mathrm{~Hz}$

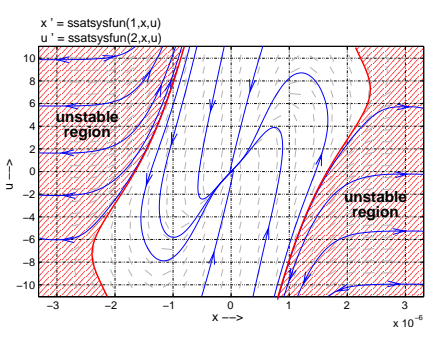

(b) System with plant output saturation

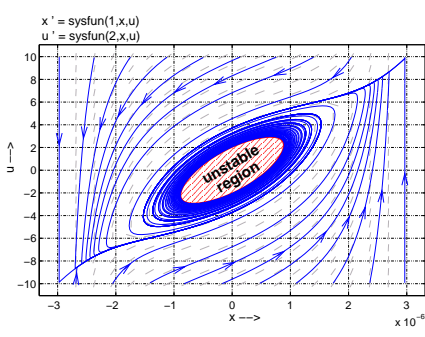

(d) Unstable equilibrium point
Fig. 5. Phase portrait of the mechanical model incorporated with the inverse controller

into a very bad closed-loop dynamics, but does not change the stability of the system. An other case is shown in Fig. 5(d), where $\omega_{c}$ has crossed the pole location. The equilibrium point is in that case unstable (invalid inequality (9)), but the nonlinear system results into a limited cycle.

\section{CONCLUSiOnS}

The stability has been graphically tested for one particular control adjustment with Lyapunov's direct and indirect method. The used function candidate proves the local stability in a wide area.

The system stability is guaranteed within the maximal applicable signals as long as the equilibrium point is asymptotically stable. A Limitation in the maximal detectable displacement limits the system stability.

Since one cannot conclude stability for some other control adjustments, more analysis and also practical tests have to be done. Another task is finding a Lyapunov function candidate, which reproduces the gained phase plane results better.

\section{REFERENCES}

[1] Van Kampen, R. P., M. J. Vellekoop, P. M. Sarro, and R. F. WOLFENBUTTEL: Application of electrostatic feedback to critical damping of an integrated silicon capacitive accelerometer. Sensors and Actuators A, 43:100-106, 1994.

[2] Lu, Crist, Mark Lemkin, and Bernhard E. Boser: A monolithic surface micromachined accelerometer with digital output. IEEE Journal of Solid-State Circuits, 30(12):1367-1373, December 1995.

[3] LEMKIN, MARK and BERNHARD E. BOSER: Three-axis micromachined accelerometer with a cmos position-sense interface and digital offsettrim electronics. IEEE Journal of Solid-State Circuits, 34(4):456-468, April 1999.
[4] Handtmann, MaRtin: Dynamische Regelung mikroelektromechanischer Systeme (MEMS) mit Hilfe kapazitiver Signalwandlung und Kraftrückkoppelung. Doktorarbeit, Technische Universität München, München, Juli 2004. erschienen unter Shaker Verlag Aachen, 2005.

[5] Wine, David W., Mark P. Helsel, Lorne Jenkins, Hakan URey, and THOR D. OSBORN: Performance of a biaxial mems-based scanner for microdisplay applications. In MOTAMEDI, M. EDWARD and ROLF GOERING (editors): MOEMS and Miniaturized Systems, volume 4178 of Proceedings of SPIE, pages 186-196, Santa Clara, CA, September 2000. SPIE.

[6] Horsley, David A., NAiYavudhi WongKomet, Roberto Horowitz, and AlBert P. PISANO: Precision positioning using a microfabricated electrostatic actuator. IEEE Transactions on Magnetics, 35(2):993-999, March 1999.

[7] Cheung, Patrick, Roberto Horowitz, and Roger T. Howe: Design, fabrication, position sensing, and control of an electrostaticallydriven polysilicon microactuator. IEEE Transactions on Magnetics, 32(1):122-128, January 1996.

[8] LiaO, Ke-Min, Yi-ChiH WANG, ChiH-Hsien YeH, and Rongshun CHEN: Closed-loop adaptive control for torsional micromirrors. In El-FATATRY, AYMAN (editor): MOEMS and Miniaturized Systems IV, volume 5346 of Proceedings of SPIE, pages 184-192, Bellingham, WA, 2004. SPIE.

[9] Park, Sungsu, Roberto Horowitz, and Chin-Woo Tan: Adaptive controller design of mems gyroscopes. In Proceedings of the 2001 IEEE Intelligent Transportation Systems, pages 496-501, Oakland, CA, August 25-29, 2001. IEEE.

[10] PARK, SUNGSU and RobERTo Horowitz: Adaptive control for the conventional mode of operation of mems gyroscopes. Journal of Microelectromechanical Systems, 12(1):101-108, February 2003.

[11] Sane, Harshad S., Navid Yazdi, and Carlos Mastrangelo: Robust control of electrostatic torsional micromirrors using adaptive sliding-mode control. In Proceedings of SPIE - Photonics West 2005 , volume 5719, pages 115-126, San Jose, CA, January 22-27, 2005. SPIE.

[12] Gaura, E. I., N. Ferreira, R. J. Rider, and N. Steele: Closedloop, neural network controlled accelerometer design. In LAUDON, MATTHEW and BART ROMANOWICZ (editors): Proceedings of the Third International Conference on Modeling and Simulation of Microsystems, pages 513-516, San Diego, CA, March 27-29, 2000.

[13] KrAft, M., C. P. Lewis, and T. G. Hesketh: Closed-loop silicon accelerometers. IEE Proceedings on Circuits, Devices and Systems, 145(5):325-331, October 1998.

[14] Lu, MichaEL S.-C. and GARY K. FEDDER: Position control of parallel-plate microactuators for probe-based data storage. Journal of Microelectromechanical Systems, 13(5):759-769, October 2004.

[15] BRyzeK, JANusz, ERIC ABbott, Anthony Flannery, David CAGle, and JaCEK Maitan: Control issues for mems. In Proceedings of the $42^{\text {nd }}$ IEEE Conference on Decision and Control, volume 3, pages 3039-3047, Maui, Hawaii, December 9-12, 2003. IEEE.

[16] Wolfram, Heiko, Ralf Schmiedel, Karla Hiller, Torsten Aurich, Wolfgang GÜNTHER, StefFen Kurth, JAN MEHNER, WOLFRAM DÖTZEL, and THOMAS GESSNER: Model building, control design and practical implementation of a high precision, high dynamical mems acceleration sensor. In CANÉ, CARLES, JUNG-CHIH CHIAO, and Fernando Vidal Verdú (editors): Smart Sensors, Actuators, and MEMS II, volume 5836 of Proceedings of SPIE, pages 326-340, Sevilla, Spain, May 09-11, 2005. SPIE.

[17] Bode, HENDRIK W.: Network Analysis and Feedback Amplifier Design. D. van Nostrand Company, Inc., Princeton, NJ, 1945.

[18] Skogestad, Sigurd and IAn Postlethwaite: Multivariable Feedback Control - Analysis and Design. John Wiley \& Sons Ltd. Chichester, England, 1996.

[19] ÅströM, KARL J.: Limitations on control system performance. European Journal of Control, 6(1):2-20, 2000.

[20] ARnold, DAVID and John C. POLKIng: Ordinary Differential Equations Using Matlab. Pearson Higher Education, 2 edition, 1999. 